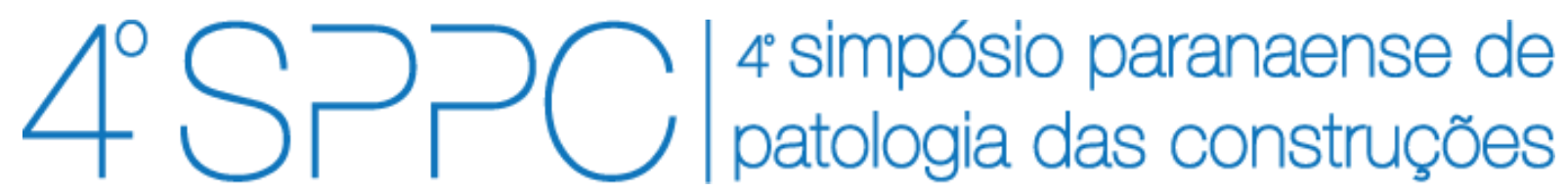

ISSN 2526-7248 artigo n. 4SPPC131, pp. 291-299, 2019

\title{
Influência do ativador alcalino no pH e resistência à compressão do cimento supersulfatado: estudo exploratório para uso no concreto armado
}

\author{
Schuster, Gecieli ${ }^{1}$; Homrich, Jefferson Teixeira Olea ${ }^{2}$; Stedile, Keila ${ }^{3}$; Angulski da Luz, \\ Caroline $^{4}$; Medeiros, Marcelo Henrique Farias ${ }^{5}$ \\ ${ }^{1}$ Mestranda, Universidade Tecnológica Federal do Paraná, gecieli.schuster@hotmail.com \\ ${ }^{2}$ Professor Me., Faculdade de Pato Branco (FADEP), jefferson@fadep.br \\ ${ }^{3}$ Graduanda em Engenharia Civil., Universidade Tecnológica Federal do Paraná, \\ kstedile@alunos.utfpr.edu.brm \\ ${ }^{4}$ Professora Drª ., Universidade Tecnológica Federal do Paraná, algulski@hotmail.com \\ ${ }^{5}$ Professor Dr., Universidade Federal do Paraná, medeiros.ufpr@gmail.com
}

Resumo: $\mathrm{O}$ consumo excessivo de recursos naturais e a grande liberação de $\mathrm{CO}_{2}$ na produção de cimento Portland (CP) vem aumentando a necessidade de se encontrar cimentos com materiais alternativos em sua produção. O cimento supersulfatado aparece como uma opção ao CP, produzido a partir da mistura de escória de alto forno, uma fonte de sulfato de cálcio e um ativador alcalino. Diversos estudos sobre as propriedades deste cimento vêm sendo realizadas, porém sua durabilidade é pouco conhecida. Pensando nisso, este estudo busca analisar a influência do ativador alcalino no $\mathrm{pH}$ e resistência à compressão deste aglomerante, com vistas a sua futura aplicação em concreto armado. Para isso, foram utilizados cimentos Supersulfatados com $\mathrm{KOH}$ e $\mathrm{NaOH}$ como ativadores alcalinos em 3 teores diferentes $(0,2,0,5$ e $0,8 \%)$. Para resistência à compressão medida em pasta, ambos os ativadores apresentaram valores próximos e compatíveis com diversas aplicações para teor de $0,5 \%$ de ativador. Além disso, os ensaios de $\mathrm{pH}$ mostraram que há influência do ativador neste parâmetro, e apenas o teor de $0,2 \%$ apresentou valor abaixo do indicado como ideal para formação da película passivante.

Palavras-chave: Cimento Supersulfatado, resistência mecânica, concreto armado,

Abstract: The excessive consumption of natural resources and the large release of $\mathrm{CO}_{2}$ in the Portland cement (CP) production has increased the need to find cements with alternative materials in their production. Supersulphated cement appears as an option to CP, produced from blending furnace slag, a source of calcium sulphate and an alkaline activator. Several studies on the properties of this cement have been carried out, however the viability of its use is still unknown. This study aims to analyze the influence of the alkaline activator on the $\mathrm{pH}$ and compressive strength of this binder, with a view to its application in reinforced concrete. For this, Supersulphated cements with $\mathrm{KOH}$ and $\mathrm{NaOH}$ were used as alkaline activators, with 3 different contents (0.2, 0.5 and $0.8 \%)$. For compressive strength measured in paste, both activators presented close and possible values of application in reinforced concrete to $0.5 \%$ activator content. The $\mathrm{pH}$ tests showed that there is an influence of the activator in this parameter, and only the content of $0.2 \%$ presented below ideal value for application in reinforced concrete.

Keywords: Supersulphated cement, reinforced concrete, compressive strength 
SCHUSTER, G.; HOMRICH, J. T. O.; STEDILE, K.; ANGULSKI DA LUZ, C.; MEDEIROS, M. H. F.; INFLUÊNCIA DO ATIVADOR ALCALINO NO PH E RESISTÊNCIA À COMPRESSÃO DO CIMENTO SUPERSULFATADO: ESTUDO EXPLORATÓRIO PARA USO NO CONCRETO ARMADO. 40 Simpósio Paranaense de Patologia das Construções (40 SPPC), artigo 4SPPC131, pp. 291 - $299,2019$.

\section{Introdução} DOI: $10.4322 / 2526-7248.051$

Com o consumo excessivo de recursos naturais e a grande liberação de $\mathrm{CO}_{2}$ na produção do Cimento Portland $(\mathrm{CP})$, os estudos para encontrar materiais alternativos na produção de novos cimentos vêm aumentando gradativamente. Um cimento que utiliza materiais alternativos é o cimento supersulfatado (CSS), que utiliza subprodutos para a sua produção, como a escória de alto forno, e uma fonte de sulfato de cálcio [1-2].

A produção do CSS tem a vantagem de ser simples e de baixo custo, sendo este um ligante livre ou quase livre de clinquer [3]. Para a fabricação do CSS é necessário ter 80 a $85 \%$ de escória e 10 a $15 \%$ de uma fonte de sulfato de cálcio, podendo acrescentar um ativador alcalino, como o $\mathrm{KOH}, \mathrm{NaOH}, \mathrm{CPV}$ e $\mathrm{Ca}(\mathrm{OH})_{2}$, com proporções variáveis [4].

Quando um ativador é adicionado na produção do CSS, este auxilia na hidratação do cimento, atacando o grão de escória e fazendo com que a produção dos produtos hidratados ocorra com maior rapidez. Porém os ativadores afetam o $\mathrm{pH}$ do CSS, que nas primeiras horas de hidratação chega a ser 12,5 tendendo a se estabilizar posteriormente. Entretanto, quando o pH é maior que 13, a etringita pode não se formar ou até mesmo ficar instável, podendo, se recombinar com outros compostos. Este efeito pode causar a queda de resistência à compressão [3-5].

A reação de hidratação do CSS depende do tipo e proporção de ativador. Na Equação 1 tem-se o processo de hidratação do CSS de forma simplificada.

$$
\mathrm{C}_{5} \mathrm{~S}_{3} \mathrm{~A}+\mathrm{CH}+3 \mathrm{C} \overline{\mathrm{S}}+34 \mathrm{H} \rightarrow \mathrm{C}_{6} \mathrm{~A}_{3} \mathrm{H}_{32}+3 \mathrm{CSH}
$$

Na reação é possível observar que o principal componente da escória representada pela gehlenita $\left(\mathrm{C}_{5} \mathrm{~S}_{3} \mathrm{~A}\right)$, reage com o ativador alcalino (hidróxido de cálcio $(\mathrm{CH})$ ) juntamente com a fonte de cálcio $(\mathrm{CS})$ e água $(\mathrm{H})$ formando a etringita (até 7 dias) e o CSH que surge após 2-3 dias [3-6].

Diversos estudos sobre este aglomerante vêm sendo desenvolvidos nos últimos tempos, porém nenhum com vistas a sua aplicação no concreto armado. Ao ser utilizado para este fim, o mesmo deve, além de outras características, apresentar determinada resistência mecânica e um pH alcalino, pois nesse caso, o concreto funciona como uma proteção física e química ao aço contra agentes agressivos. Em relação a resistência à compressão, é possível obter concretos autoadensáveis com CSS e atingir resistências com valores próximos à $40 \mathrm{MPa}$ aos 28 dias de hidratação, valores que tornam sua aplicação possível para diversos fins [7].

Com relação a barreira química que o concreto exerce, para a aplicação deste aglomerante em concreto armado é necessário que haja possibilidade do mesmo formar a camada passivadora no entorno do aço. Esta é entendida como a resistência à corrosão proporcionada por uma película fina de um filme de óxido estável e aderente formado na superfície do metal [8-9]. 
SCHUSTER, G.; HOMRICH, J. T. O.; STEDILE, K.; ANGULSKI DA LUZ, C.; MEDEIROS, M. H. F.; INFLUÊNCIA DO ATIVADOR ALCALINO NO PH E RESISTÊNCIA À COMPRESSÃO DO CIMENTO SUPERSULFATADO: ESTUDO EXPLORATÓRIO PARA USO NO CONCRETO ARMADO. $4^{\circ}$ Simpósio Paranaense de Patologia das Construções (40 SPPC), artigo 4SPPC131, pp. 291 - $299,2019$. DOI: $10.4322 / 2526-7248.051$

A formação da película passivadora ocorre com valores de $\mathrm{pH}$ do concreto entre 10 e 13 e sob essas condições, uma fina camada de óxidos é formada, composta por $\mathrm{Fe}_{2} \mathrm{O}_{3}$ e/ou $\mathrm{Fe}_{3} \mathrm{O}_{4}$, ou ainda pela presença de $\gamma-\mathrm{FeOOH}$, sendo regulada principalmente pela disponibilidade de oxigênio e pelo $\mathrm{pH}$ na interface aço/concreto [10-11-12].

É necessário levar em consideração que o CSS deve apresentar valores de pH entre 11,5 e 12,2 para formação de cristais de etringita, principal responsável pela resistência nas matrizes de CSS e, além disso, amostras de CSS dosadas com diferentes teores de $\mathrm{KOH}$ chegaram a valores de $\mathrm{pH}$ acima de 11,5 [13-14-15]. Estes valores seriam compatíveis com os apresentados pelo cimento Portland, possibilitando a formação do ambiente alcalino necessária à passivação do aço

Diante do exposto, este trabalho teve como objetivo analisar a influência do ativador alcalino no $\mathrm{pH}$ e na resistência mecânica do CSS, para fins de aplicação em estruturas de concreto armado.

\section{Metodologia}

Para realização desta pesquisa, a metodologia foi dividida em duas etapas, sendo a primeira a apresentação e caracterização dos materiais utilizados, e a segunda contempla os ensaios realizados para atingir os objetivos da pesquisa.

\subsection{Materiais}

O cimento supersulfatado utilizado foi obtido com $85 \%$ de escória de alto-forno e 15 $\%$ de anidrita, variando-se as proporções dos ativadores alcalinos. Para este último, foram utilizados neste trabalho, o hidróxido de potássio $(\mathrm{KOH})$ e hidróxido de sódio $(\mathrm{NaOH})$, com proporção de 0,2; 0,5 e 0,8 \%.

Inicialmente o material passou por secagem em estufa durante 24 horas, em temperatura controlada de $105^{\circ} \mathrm{C}$. Após esta estapa, a escória foi submetida à moagem por 3 horas em moinho de bolas da marca Servitech, modelo CT 242, até chegar ao valor de $5.087 \mathrm{~cm}^{2} / \mathrm{g}$ de superfície blaine, de acordo com ensaio realizado seguindo a NBR NM 76 [16].

Após o preparo, o material passou por análise granulométrica à laser, difratogrametria por raios $X(D R X)$ e análise química por fluorescência de raios $X$ (FRX). A distribuição granulométrica da escória, conforme Figura 1, apresenta $10 \%$ das partículas passantes em malha de 1,64 $\mu \mathrm{m}$ de abertura, $50 \%$ em malha de $18,50 \mu \mathrm{m}, 90 \%$ em malha de $62,23 \mu \mathrm{m}$ e apresentou um diâmetro médio de 26,16 $\mu \mathrm{m}$.

A composição química da escória é apresentada na Tabela 1, onde é possível observar que a escória está de acordo com os parâmetros especificados na norma europeia [17] para a utilização em CSS.

Tabela 1: Composição química da escória por FRX.

\begin{tabular}{ccccccccc}
\hline Óxidos & $\mathrm{CaO}$ & $\mathrm{SiO}_{2}$ & $\mathrm{Al}_{2} \mathrm{O}_{3}$ & $\mathrm{MgO}$ & $\mathrm{SO}_{3}$ & Outros & $\mathrm{CaO}+\mathrm{MgO}+\mathrm{SiO}_{2}$ & $(\mathrm{CaO}+\mathrm{MgO}) / \mathrm{SiO}$ \\
\hline Composição & $47,50 \%$ & $34,70 \%$ & $8,90 \%$ & $4,30 \%$ & $1,60 \%$ & $2,90 \%$ & 86,27 & 1,49 \\
\hline EN 15743:2010 & - & - & - & - & - & - & $\geq 66,7$ & $\geq 1,0$
\end{tabular}


SCHUSTER, G.; HOMRICH, J. T. O.; STEDILE, K.; ANGULSKI DA LUZ, C.; MEDEIROS, M. H. F.; INFLUÊNCIA DO ATIVADOR ALCALINO NO PH E RESISTÊNCIA À COMPRESSÃO DO CIMENTO SUPERSULFATADO: ESTUDO EXPLORATÓRIO PARA USO NO CONCRETO ARMADO. 40 Simpósio Paranaense de Patologia das Construções (40 SPPC), artigo 4SPPC131, pp. 291 - $299,2019$. DOI: $10.4322 / 2526-7248.051$

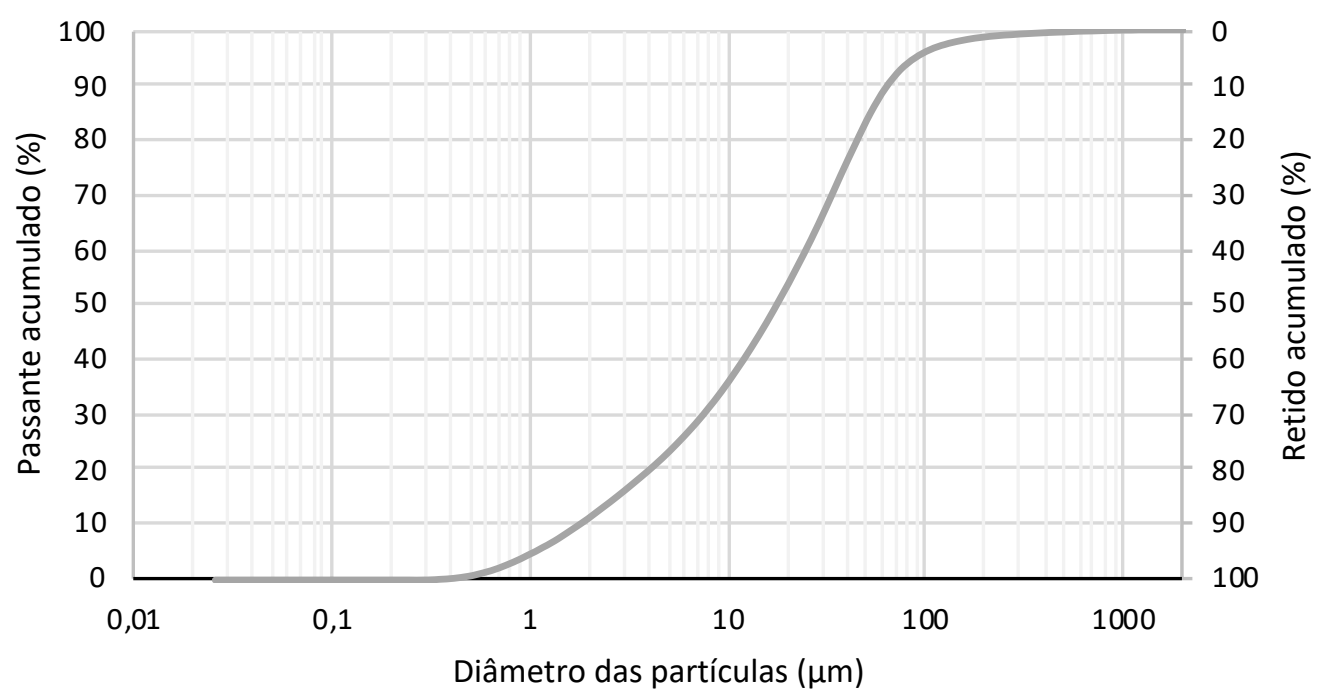

Figura 1: Curva granulométrica da escória

Por meio da técnica de difração de raios $X(D R X)$, obteve-se o difratograma da escória utilizada neste trabalho, apresentado na Figura 2. O material apresenta um comportamento amorfo, pois verifica-se que não há presença de picos de cristalinidade característicos, fator que aumenta a empregabilidade da escória para a produção de CSS.

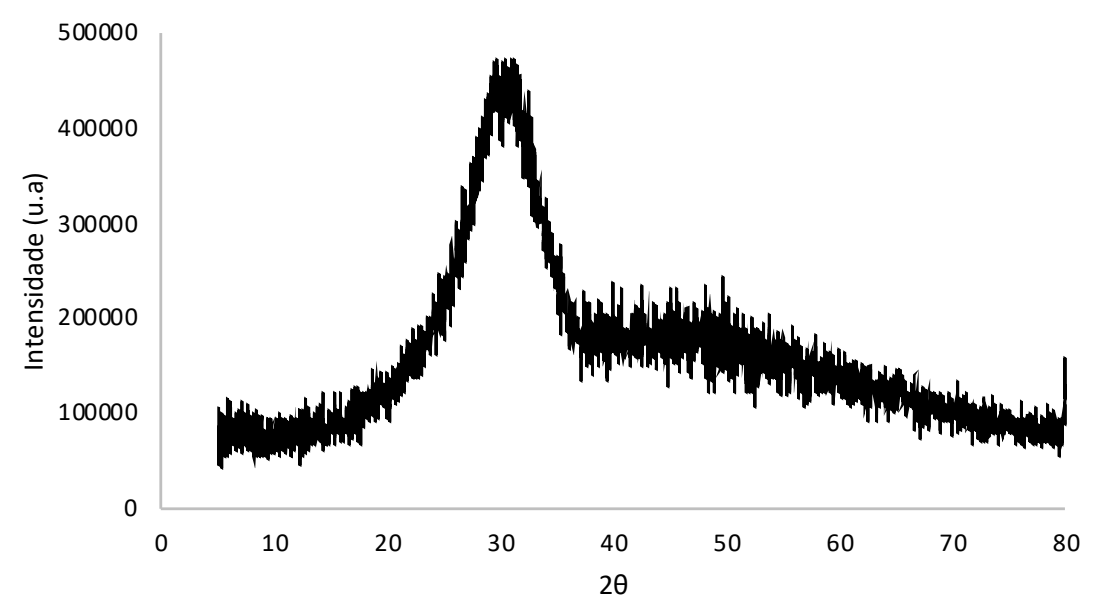

Figura 2: DRX da escória granulada de alto forno.

A fonte de sulfato de cálcio utilizada foi obtida aravés da calcinação da gipsita em mufla, à $650^{\circ} \mathrm{C}$, por uma hora, a uma taxa de aquecimento de $50^{\circ} \mathrm{C} / \mathrm{min}$, originando anidrita.

\subsection{Métodos}

Para análise da influência dos ativadores alcalinos nas medidas de $\mathrm{pH}$ e resistência mecânica, foram dosadas pastas com CSS onde se utilizou $\mathrm{NaOH}$ como ativador e outra dosagem em que se produziu o cimento com $\mathrm{KOH}$, sendo que para cada um dos ativadores, três teores diferentes foram testados $(0,2 \%, 0,5 \%$ e $0,8 \%)$. 0 procedimento para o preparo das pastas de cimento é descrito a seguir: 
SCHUSTER, G.; HOMRICH, J. T. O.; STEDILE, K.; ANGULSKI DA LUZ, C.; MEDEIROS, M. H. F.; INFLUÊNCIA DO ATIVADOR ALCALINO NO PH E RESISTÊNCIA À COMPRESSÃO DO CIMENTO SUPERSULFATADO: ESTUDO EXPLORATÓRIO PARA USO NO CONCRETO ARMADO. $4^{\circ}$ Simpósio Paranaense de Patologia das Construções (40 SPPC), artigo 4SPPC131, pp. 291 - $299,2019$. DOI: $10.4322 / 2526-7248.051$

Etapa 1 - Mistura dos componentes secos: pesagem e homogeneização das proporções pré-definidas de escória e anidrita.

Etapa 2 - Preparo da solução dos ativadores alcalinos, pela dissolução dos mesmos em água, mantendo a relação água/cimento 0,4.

Etapa 3 - Mistura dos componentes sólidos e líquidos: após pesagem dos reagentes, a porção sólida (escória e anidrita) foi cuidadosamente adicionada à porção líquida (água e ativadores alcalinos) com agitação manual por 60 segundos.

Etapa 4 - Moldagem e desmoldagem: após completa homogeneização, as pastas foram moldadas e identificadas, em moldes com $20 \mathrm{~mm}$ de diâmetro x $40 \mathrm{~mm}$ de altura, sendo estes acondicionados em câmera úmida e ambiente climatizado sob temperatura constante de $23^{\circ} \mathrm{C}$. Passadas 24 horas, as amostras foram desmoldadas. As amostras permaneceram na câmara, com cura submersa em água, até as idades de ensaio.

A determinação dos valores do $\mathrm{pH}$ foi realizada nas amostras após a etapa 3, as medidas foram feitas diariamente nos primeiros 7 dias e posteriormente um acompanhamento semanal até o período de 90 dias. $\mathrm{O} \mathrm{pH}$ foi aferido diretamente na água na qual as amostras estavam submersas com o auxílio de um medidor de $\mathrm{pH}$ marca AKSO, modelo AK90 [15]. A água da cura não foi trocada durante todo o período de análise.

Os ensaios de resistência à compressão foram realizados com os corpos de prova moldados na etapa 4 [4], utilizando uma prensa da marca EMIC, modelo DL-30000, com uma célula de carga de $300 \mathrm{kN}$, nas idades de 7, 28 e 90 dias realizados em triplicatas. Os resultados apresentados são uma média dos 3 corpos de prova.

\section{Resultados}

\subsection{Resistências à Compressão}

Na Figura 3, têm-se os resultados obtidos das resistências à compressão, onde é possível avaliar o comportamento dos ativadores alcalinos em diferentes concentrações. Para isso, o teste foi realizado com idades de hidratação de 7, 28 e 90 dias.
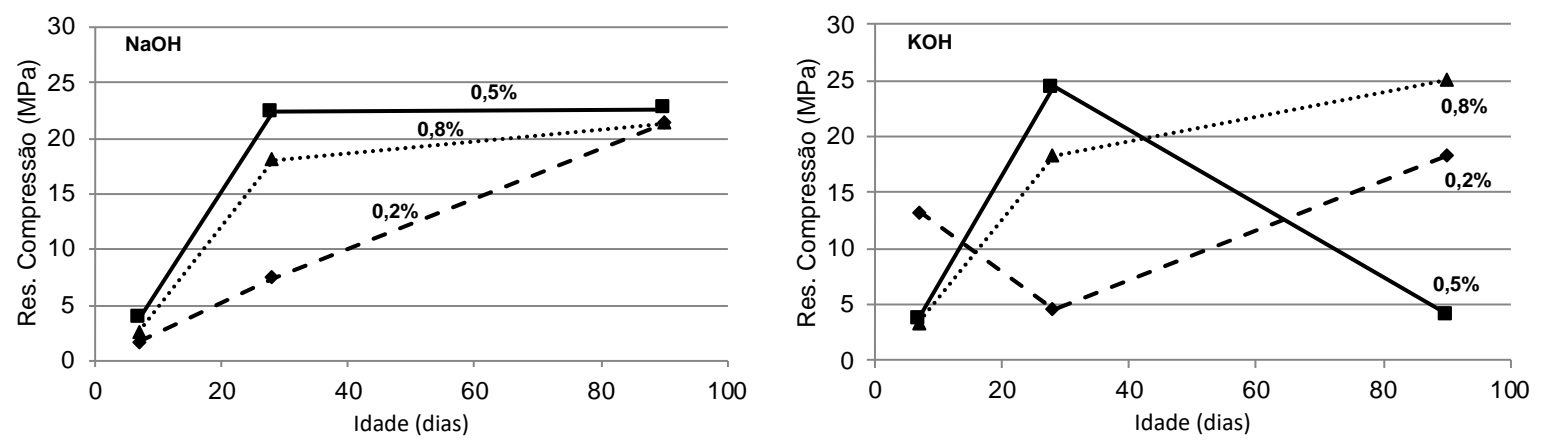

Figura 3: Resistência à compressão ao longo da hidratação 
SCHUSTER, G.; HOMRICH, J. T. O.; STEDILE, K.; ANGULSKI DA LUZ, C.; MEDEIROS, M. H. F.; INFLUÊNCIA DO ATIVADOR ALCALINO NO PH E RESISTÊNCIA À COMPRESSÃO DO CIMENTO SUPERSULFATADO: ESTUDO EXPLORATÓRIO PARA USO NO CONCRETO ARMADO. $4^{\circ}$ Simpósio Paranaense de Patologia das Construções (40 SPPC), artigo 4SPPC131, pp. 291 - $299,2019$. DOI: $10.4322 / 2526-7248.051$

$\mathrm{Na}$ Figura 3, pode-se observar que as amostras com ativador $\mathrm{NaOH}$ apresentaram resistência à compressão menores que $5 \mathrm{MPa}$ aos 7 dias, com uma evolução nos valores ao longo da hidratação, chegando a resistência acima de $20 \mathrm{MPa}$ para 90 dias. Os baixos valores de resistência na idade inicial é causado pelo baixo teor de alumina apresentado pela escória utilizada, que chega próximo às escórias de baixa reatividade, e o ganho de resistência mais lento apresentado pela amostra com 0,2 \% pode ser devido à taxa de hidratação ser menor para CSS com baixos teores de ativador [4].

Ao aplicar uma análise de variância (ANOVA), com nível de significância de $5 \%$, é possível afirmar que só existe diferença estatística na idade de 28 dias e para amostra de $0,2 \%$, ou seja, aos 7 e 90 dias, não existe diferença estatística entre as 3 amostras e aos 28 dias as amostras com 0,5\% e 0,8 \% são estatisticamente iguais.

Ainda conforme a Figura 3, também é possível observar que, para o ativador $\mathrm{KOH}$, os teores com $0,5 \%$ e $0,8 \%$ apresentam valores de resistência inferiores a $5 \mathrm{MPa}$ aos 7 dias, evoluindo para 24,4 $\mathrm{MPa}$ e 18,3 $\mathrm{MPa}$, respectivamente. Este comportamento na idade inicial é semelhante ao encontrado em pesquisas correlatas [18] e pode ser ocasionado pelo isolamento dos grãos de escória, causado pelo acúmulo de agulhas de etringita no seu entorno, diante de alto teor de álcalis para teores de sulfato maiores [13]. Ao contrário, a amostra com 0,2\%, decresceu de 13,3 $\mathrm{MPa}$ aos 7 dias para 4,6 $\mathrm{MPa}$ aos 28 dias, causado, provavelmente, pela transformação da etringita em monossulfato [5].

Quando se trata da evolução de resistência entre 28 e 90 dias, há crescimento nos valores das pastas com 0,8 \% e 0,2\%, comportamento contrário ao da amostra com $0,5 \%$, que apresenta redução em sua resistência para valores inferiores a 5,0 MPa. Uma possibilidade para esta redução nas pastas com $0,5 \%$ é a mesma citada para comportamento entre os 7 e 28 dias da amostra com 0,2\% de ativador, a instabilidade da etringita, sendo transformada em monossulfato, quando há carência de sulfato de cálcio [5].

Através da análise de variância (ANOVA), com nível de significância de $5 \%$, é possível afirmar que, aos 7 dias não existe diferença estatística entre as amostras analisadas, porém aos 28 dias, as pastas com 0,2 \% são estatisticamente diferentes das demais. Já para os 90 dias de hidratação, a amostra que contém teor de 0,5\% apresenta diferença estatística para as demais. Em pesquisa utilizando os mesmos materiais para CSS, o comportamento nas 3 idades é diferente, pois foram encontrados valores maiores de resistência para o teor de $0,2 \%$ de ativador, juntamente com o $0,5 \%$, e os menores valores foram com $0,8 \%$ de $\mathrm{KOH}$ [18].

\subsection{Monitoramento do pH}

$\mathrm{Na}$ Figura 4, são apresentados os valores de monitoramento do $\mathrm{pH}$, que ocorreu durante os 90 dias de hidratação do CSS, para ambos os ativadores alcalinos utilizados. $\mathrm{O}$ pH do cimento supersulfatado deve ter teor alcalino entre 11 até 12 para que assim possa ocorrer um processo de hidratação. Quando esses valores de $\mathrm{pH}$ são maiores que 12, pode ocorrer a dissolução da etringita e assim afetar a resistência à compressão [3-5]. 

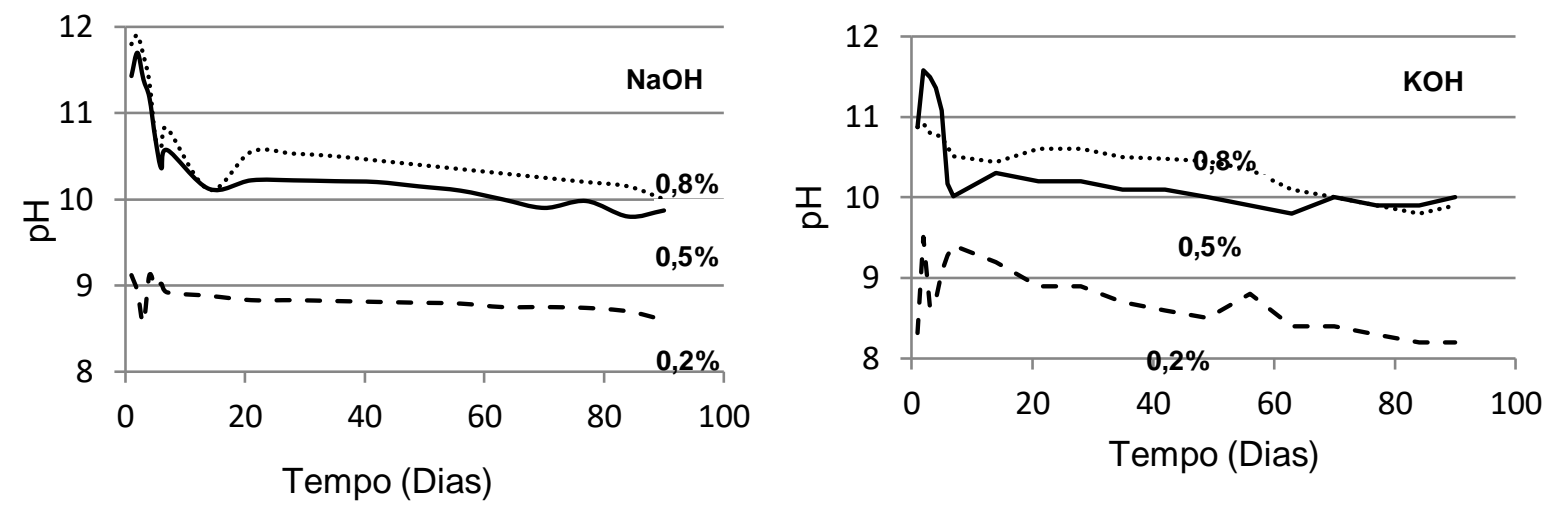

Figura 4: Monitoramento de pH utilizando o $\mathrm{NaOH}$ (esquerda) e $\mathrm{KOH}$ (direita) como ativador.

$\mathrm{O}$ ativador $\mathrm{NaOH}$ obteve valores de $\mathrm{pH}$ abaixo do que seria ideal para o teor de 0,2 $\%$, com pH 8,8 na idade de 28 dias e depois estabilizou esse valor, o que é possível explicar os baixos valores da resistência [13-14]. Pode-se notar que o teor de ativador teve influencia nos valores de $\mathrm{pH}$, sendo que para os 0,5 e $0,8 \%$ o $\mathrm{pH}$ nos primeiros dias de hidratação foi de 11,8 e após esta idade diminuiu para 10,2, estabilizando-se nesses valores até os 90 dias de hidratação, ficando assim com 0 $\mathrm{pH}$ alcalino.

$\mathrm{O}$ ativador $\mathrm{KOH}$ apresentou um comportamento parecido com o $\mathrm{NaOH}$, no qual com teor de $0,2 \%$ o pH ficou abaixo do ideal [13-14], e ao aumentar o teor de ativador, o $\mathrm{pH}$ demonstrou um valor mais alcalino. Onde, para o teor de 0,5\%, nos primeiros dias de hidratação, o seu pH foi de 11,6 e, aos 28 dias, de 10,2. Já para 0,8\%, as medidas iniciais apresentaram valores próximos à 11,0 e, aos 28 dias, pequena redução, chegando em 10,6. Após os 28 dias, as amostras com maior teor de ativador estabilizaram-se constantes ao longo das medições, porém a com menor dosagem de $\mathrm{KOH}$ apresentou queda para valores próximos à 8,0.

Os valores encontrados de $\mathrm{pH}$ para as 3 pastas com $\mathrm{KOH}$ são explicados pois este ativador tende a apresentar maiores valores de $\mathrm{pH}$ nas primeiras horas de hidratação [2]. Porém, são inferiores aos encontrados em pesquisa que utilizou os mesmos teores de ativador, com dosagem diferente de escória e anidrita [15].

Para os dois ativadores analisados, as amostras apresentaram comportamento semelhante nos valores de $\mathrm{pH}$ ao longo da hidratação quando se compara com resultados da bibliografia [15].

\section{Conclusões}

Este trabalho experimental permite elaborar as seguintes conclusões:

- Para os valores de resistência à compressão, houve pouca influência com a mudança do tipo ativador alcalino $(\mathrm{NaOH}$ e $\mathrm{KOH})$. As amostras com $\mathrm{KOH}$ se mostraram mais sensíveis à influência do teor de ativador alcalino. 
SCHUSTER, G.; HOMRICH, J. T. O.; STEDILE, K.; ANGULSKI DA LUZ, C.; MEDEIROS, M. H. F.; INFLUÊNCIA DO ATIVADOR ALCALINO NO PH E RESISTÊNCIA À COMPRESSÃO DO CIMENTO SUPERSULFATADO: ESTUDO EXPLORATÓRIO PARA USO NO CONCRETO ARMADO. 40 Simpósio Paranaense de Patologia das Construções (40 SPPC), artigo 4SPPC131, pp. 291 - $299,2019$. DOI: $10.4322 / 2526-7248.051$

- Somente os teores de 0,5\% apresentaram valores acima de $20 \mathrm{MPa}$, aos 28 dias, aplicável em concreto armado de acordo com a NBR 6118 [19].

- Foi possível observar que existe influência do tipo de ativador alcalino nos valores de $\mathrm{pH}$. Além disso, há possibilidade de aplicação do CSS em concreto, já que os valores apresentados para ambos os ativadores, o pH está acima dos valores informados como ideiais para formação da película passivadora.

- As amostras apresentaram pH com valores abaixo do ideal para concreto armado, foram as que utilizaram teores de $0,2 \%$ de ativador alcalino.

- Análises aprofundadas sobre o tempo de passivação e a estabilidade da película ao longo das idades do concreto devem ser realizadas para que haja confirmação de viabilidade de seu uso em termos de capacidade de proteção do aço carbono.

\section{Agradecimentos}

Os autores agradecem a infraestrutura e o suporte fornecidos pela Universidade Técnológica Federal do Paraná (UTFPR), câmpus Pato Branco, ao Programa de Pós- Graduação em Engenharia de Construção Civil (PPGECC) da Universidade Federal do Paraná (UFPR) e ao Programa de Pós-Graduação em Tecnologia de Processos Químicos e Bioquímicos (PPGTP) da UTFPR.

\section{Referências}

[1] HORSLEY, C.; EMMERT, M. H.; SAKULICH, A. (2016) Influence of alternative fuels on trace element content of ordinary portland cement. Fuel. 481-489.

[2] RESCHKE, J. S. (2003) Escória Granulada De Fundição Utilizada Como Substituição Ao Cimento Em Concretos. Dissertação (mestrado), UFRGS, Porto Alegre-RS.

[3] JUENGER, M. C. G. et al. (2011) Advances ins alternative cementitious binders. Cement and Concrete Research. 1232-1243.

[4] GRUSKOVNJAK, A. et al. (2008) Hidration mechanisms of super sulphates slag cement. Cement and Concrete Reasearch. 983-992.

[5] GRACIOLI, B. (2017) Considerations on the mechanical behavior and hydration process supersulfated cement (CSS) formulated with phosphogypsum. Matéria, Rio de Janeiro.

[6] ANGULSKI DA LUZ, C.; HOOTON, R. D. (2015) Influence of curing temperature on the process of hydration of supersulfated cements at early age. Cement and concrete research. 69-75.

[7] HOMRICH, J. T. O. (2018) Avaliação do comportamento do cimento supersulfatado em concreto autoadensável. Dissertação (mestrado), UTFPR, Pato Branco-PR. 
SCHUSTER, G.; HOMRICH, J. T. O.; STEDILE, K.; ANGULSKI DA LUZ, C.; MEDEIROS, M. H. F.; INFLUÊNCIA DO ATIVADOR ALCALINO NO PH E RESISTÊNCIA À COMPRESSÃO DO CIMENTO SUPERSULFATADO: ESTUDO EXPLORATÓRIO PARA USO NO CONCRETO ARMADO. 40 Simpósio Paranaense de Patologia das Construções (40 SPPC), artigo 4SPPC131, pp. 291 - $299,2019$. DOI: $10.4322 / 2526-7248.051$

[8] CARMONA, T. G. Modelos de previsão da despassivação das armaduras em estruturas de concreto sujeitas a carbonatação. São Paulo: Escola Politécnica da Universidade de São Paulo, 2005.

[9] BROOMFIELD, J. P. (2007) Corrosion of Steel in Concrete: Understanding, investigation and repair. Taylor and Francis.

[10] KRUGER, J. (1989) The nature of the passive film on iron and ferrous alloys. Corrosion Science. 149-162.

[11] SATO, N. (1990) An overview on the passivity of metals. Corrosion Science. 119.

[12] HUET, B. et al. (2005) Electrochemical behavior of mild steel in concrete: Influence of $\mathrm{pH}$ and carbonate content of concrete pore solution. Electrochimica Acta. 172-180.

[13] MATSCHEI, T.; BELLMANN, F.; STARK, J. (2005) Hydration behavior of supersulphated activated slag cement. Advances in cement research. 167-178.

[14] MUN, K. J. E. A. (2007) Basic properties of non-sintering cement using phosphogypsum and waste lime as activator. Construction and Building Materials. 1342-1350.

[15] GRACIOLI, B. (2016) Influência da temperatura de calcinação do fosfogesso no desempenho de cimentos supersulfatados. Dissertação (mestrado), UTFPR, Pato Branco - PR.

[16] NBR NM 76 (1998) Cimento Portland - Determinação da finura pelo método de permeabilidade ao ar (Método de Blaine). Associação Brasileira de Normas Técnicas - ABNT, Rio de Janeiro, Brasil.

[17] EN 15743 (2010) Supersulfated cement-composition, specification and conformity criteria. European Committee For Standardization (CEN), Brussels, Belgium.

[18] RUBERT, S. (2015) Contribuição ao estudo de cimentos supersulfatados: formulação e mecanismos de hidratação. Dissertação (mestrado), UTFPR, Pato Branco - PR.

[19] NBR 6118 (2014) Projeto de estruturas de concreto - Procedimento. Associação Brasileira de Normas Técnicas - ABNT, Rio de Janeiro, Brasil. 